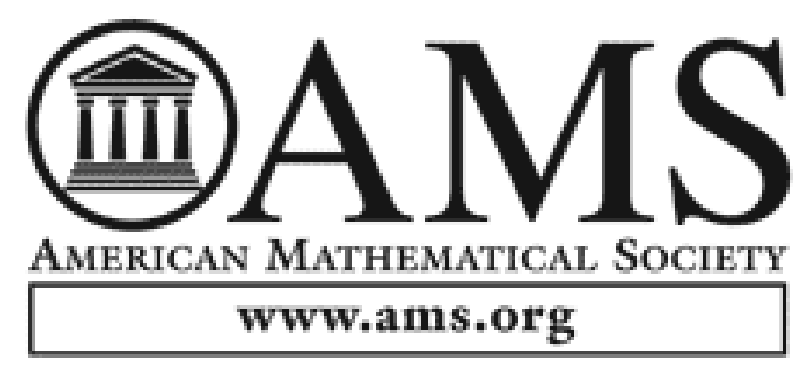

The Masses in a Symmetric Centered Solution of the n-Body Problem Author(s): Florin Nicolae Diacu

Source: Proceedings of the American Mathematical Society, Vol. 109, No. 4 (Aug., 1990), pp. 1079-1085

Published by: American Mathematical Society

Stable URL: http://www.jstor.org/stable/2048140

Accessed: $1 6 \longdiv { / 1 1 / 2 0 0 9 2 1 : 4 9 }$

Your use of the JSTOR archive indicates your acceptance of JSTOR's Terms and Conditions of Use, available at http://www.jstor.org/page/info/about/policies/terms.jsp. JSTOR's Terms and Conditions of Use provides, in part, that unless you have obtained prior permission, you may not download an entire issue of a journal or multiple copies of articles, and you may use content in the JSTOR archive only for your personal, non-commercial use.

Please contact the publisher regarding any further use of this work. Publisher contact information may be obtained at http://www.jstor.org/action/showPublisher?publisherCode=ams.

Each copy of any part of a JSTOR transmission must contain the same copyright notice that appears on the screen or printed page of such transmission.

JSTOR is a not-for-profit service that helps scholars, researchers, and students discover, use, and build upon a wide range of content in a trusted digital archive. We use information technology and tools to increase productivity and facilitate new forms of scholarship. For more information about JSTOR, please contact support@jstor.org. 


\title{
THE MASSES IN A SYMMETRIC CENTERED SOLUTION OF THE $n$-BODY PROBLEM
}

\author{
FLORIN NICOLAE DIACU
}

(Communicated by George C. Papanicolaou)

\begin{abstract}
We prove that if a planar solution of the $n$-body problem has a symmetry axis, fixed with respect to the considered frame, and the center of mass of the particle system lies on this axis during the motion, then the symmetric masses must be equal. We also show that the set of initial conditions leading to symmetric solutions has measure zero and is nowhere dense relative to the set of all initial conditions that define solutions in the space.
\end{abstract}

\section{INTRODUCTION}

We saw recently (see [4]) that if a noncollinear solution of the four body problem has a symmetry axis, then the center of mass of the particle system lies on this axis and the symmetric masses are equal. Following the proof of that paper it is easy to see that the property remains true for a solution of the $n$-body problem having two pairs of symmetric masses relative to an axis while the other $n-4$ particles belong, for all time, to this axis. Unfortunately, the method developed there cannot be used for more than two pairs of symmetric particles.

We will give here a different way to treat this problem in the case of a planar solution with any number of symmetric bodies but imposing the restriction that the baricenter of the particle system lies (at least for an open interval of time) on the symmetry axis. Such a solution will be called symmetric centered. It is actually not known (excepting the particular case with only two symmetric pairs-discussed above) if any symmetric solution with respect to an axis is always symmetric centered, but this property might be true. Anyway, the author doesn't know of any proof of this fact.

It will be shown that if a planar solution of the $n$-body problem leads to a motion that has at least one pair of symmetric particles relative to a symmetry axis, the other particles and the center of mass of the system belonging to the axis, then the symmetric particles have equal masses. In order to perform

Received by the editors February 6, 1989 and, in revised form, August 7, 1989.

1980 Mathematics Subject Classification (1985 Revision). Primary 70F10, 70F15; Secondary $34 \mathrm{C} 35,28 \mathrm{~A} 75,54 \mathrm{~F} 65$.

Key words and phrases. $n$-body problem, celestial mechanics, measure, nowhere dense set. 
the proof we will also see that along a nonsymmetric solution, the set of time moments when symmetric configurations occur is formed by isolated points.

Finally we prove that the set of initial conditions leading to symmetric centered solutions is of measure zero and nowhere dense relative to the set of all initial conditions that define solutions in $\mathbf{R}^{2}$. This means that such solutions are improbable in the sense that the set of initial conditions leading to them is poor from the measure theory and topological point of view.

\section{DEFINITIONS AND CRITERIA}

The equations of motion of the $n$-body problem, in an arbitrary fixed frame in $\mathbf{R}^{2}$, are

$$
\begin{array}{ll}
\dot{\mathbf{q}}_{\mathbf{i}}=m_{i}^{-1} \mathbf{p}_{\mathbf{i}}, & i=\overline{1, n}, \\
\dot{\mathbf{p}}_{\mathbf{i}}=\partial_{i} U(\mathbf{q}), & i=\overline{1, n},
\end{array}
$$

where $\mathbf{q}_{\mathbf{i}}=\left(q_{i}^{1}, q_{i}^{2}\right) \in \mathbf{R}^{2}, \mathbf{p}_{\mathbf{i}}=m_{i} \dot{\mathbf{q}}_{\mathbf{i}}, i=\overline{1, n}$ are the position vectors and momenta,

$$
U: \mathbf{R}^{2 n}-\Delta \rightarrow \mathbf{R}_{+}, \quad U(\mathbf{q})=\sum_{1 \leq i<j \leq n} m_{i} m_{j} /\left|\mathbf{q}_{\mathbf{i}}-\mathbf{q}_{\mathbf{j}}\right|
$$

is the potential function ( $-U(\mathbf{q})$ being the potential energy) of the system,

$$
\Delta=\bigcup_{1 \leq i<j \leq n}\left\{\mathbf{q}=\left(\mathbf{q}_{1}, \ldots, \mathbf{q}_{\mathbf{n}}\right) \in \mathbf{R}^{2 n} \mid \mathbf{q}_{\mathbf{i}}=\mathbf{q}_{\mathbf{j}}\right\}
$$

represents the collision set, $|\cdot|$ is the Euclidean norm, $\partial_{i}$ denotes the $i$ th gradient and $m_{i}>0, i=\overline{1, n}$ are the masses of the $n$ particles.

The standard results of the theory of differential equations ensure, for given initial conditions $(\mathbf{q}, \mathbf{p})(0) \in\left(\mathbf{R}^{2 n}-\Delta\right) \times \mathbf{R}^{2 n}$, the existence and uniqueness of an analytic solution $(\mathbf{q}, \mathbf{p})$ of the Equations (1), defined on a maximal interval $\left(t^{-}, t^{+}\right),-\infty \leq t^{-}<0<t^{+} \leq+\infty$. If $t^{+}$(or $t^{-}$) is finite, then the solution is said to experience a singularity at this moment.

Without loss of generality we will study the Equations (1) restricted to the invariant set $\mathbf{Q} \times \mathbf{P}$, where

$$
\mathbf{Q}=\left\{\mathbf{q} \in \mathbf{R}^{2 n} \mid \sum_{i=1}^{n} m_{i} \mathbf{q}_{\mathbf{i}}=\mathbf{0}\right\} \quad \text { and } \quad \mathbf{P}=\left\{\mathbf{p} \in \mathbf{R}^{2 n} \mid \sum_{i=1}^{n} \mathbf{p}_{\mathbf{i}}=\mathbf{0}\right\}
$$

which also means that the origin of the frame is considered in the baricenter of the particle system. This invariance is a consequence of the center of mass and momenta first integrals.

Consider $(\mathbf{q}, \mathbf{p})$ to be a solution of the Equations (1) and let $k \in \mathbf{N}, 1 \leq$ $k \leq n / 2$. Denote

$$
\mathbf{u}=\left(u_{1}, \ldots, u_{n}\right)
$$

where $u_{2 i-1}:=q_{2 i-1}^{1}-q_{2 i}^{1}, u_{2 i}:=q_{2 i-1}^{2}+q_{2 i}^{2}, i=\overline{1, k}$, and $u_{j}:=q_{j}^{2}, j=$ $\overline{2 k+1, n}$. 
Definition 1. A solution $(\mathbf{q}, \mathbf{p})$ of the Equations (1), defined on $\left(t^{-}, t^{+}\right)$, is called symmetric centered, if there exists $k \in \mathbf{N}, 1 \leq k \leq n / 2$, such that

$$
\mathbf{u}(t)=\mathbf{0}, \quad \forall t \in\left(t^{-}, t^{+}\right),
$$

where the bodies are, eventually, renumbered.

A general example (and thus the proof of the existence) of a symmetric centered solution in the $n$-body problem with equal symmetric masses is given by Wintner [6].

Since $\mathbf{u}$ is analytic the following result becomes obvious using the identity theorem of analytic functions.

Theorem 2. Let $(\mathbf{q}, \mathbf{p})$ be a solution of the Equations (1), defined on a maximal interval $\left(t^{-}, t^{+}\right)$, consider $k \in \mathbf{N}, 1 \leq k \leq n / 2$, the corresponding $\mathbf{u}$, and denote

$$
T=\left\{t \in\left(t^{-}, t^{+}\right) \mid \mathbf{u}(t)=\mathbf{0}\right\} .
$$

If $T$ has an accumulation point in $\left(t^{-}, t^{+}\right)$then the solution is symmetric centered.

An important consequence of this theorem is the following criterion.

Corollary 3. Let $(\mathbf{q}, \mathbf{p})$ be a solution of the Equations (1) and $k \in \mathbf{N}, 1 \leq k \leq$ $n / 2$, such that the corresponding $\mathbf{u}$ fulfills the conditions

$$
\mathbf{u}^{(\nu)}(0)=\mathbf{0}, \quad \forall \nu \in \mathbf{N} .
$$

Then the solution is symmetric centered.

Proof. Since $\mathbf{u}(t)=\sum_{\nu=0}^{\infty}(1 / \nu !) \mathbf{u}^{(\nu)}(0) t^{\nu}$ for $t$ in a sufficiently small interval containing 0 , it yields that $\mathbf{u}=\mathbf{0}$ on this interval and, by Theorem 2 , the conclusion follows.

\section{THE MASSES IN A SYMMETRIC CENTERED SOLUTION}

Let's now state the main result of our paper

Theorem 4. In a symmetric centered solution of the n-body problem the symmetric bodies have equal masses.

In order to perform the proof consider $M_{i}$ to be the intersection point of the straight line through $m_{i}$ that is perpendicular on the symmetry axis, and denote:

$$
\mathbf{a}_{\mathbf{i}}:=\overrightarrow{\mathrm{OM}}_{i} \text { and } \quad \mathbf{x}_{\mathbf{i}}:=\vec{M}_{i}
$$

where $P_{i}$ is the point of the plane where the particle $m_{i}$ lies. Then, obviously

$$
\mathbf{q}_{\mathbf{i}}=\mathbf{a}_{\mathbf{i}}+\mathbf{x}_{\mathbf{i}}, \quad i=\overline{1, n} .
$$

Since the Equations (1) are restricted to the invariant set $\mathbf{Q} \times \mathbf{P}$, for a symmetric centered solution of the Equations (1) we have

$$
\sum_{i=1}^{n} m_{i}\left(\mathbf{a}_{\mathbf{i}}+\mathbf{x}_{\mathbf{i}}\right)=\mathbf{0} \text {. }
$$


As $\mathbf{x}_{\mathbf{i}}=\mathbf{0}, i=\overline{2 k+1, n}$ and $\mathbf{x}_{2 \mathbf{i}-1}+\mathbf{x}_{2 \mathbf{i}}=\mathbf{0}, i=\overline{1, k}$, it follows that

$$
\sum_{i=1}^{n} m_{i} \mathbf{a}_{\mathbf{i}}+\sum_{i=1}^{k}\left(m_{2 i-1}-m_{2 i}\right) \mathbf{x}_{2 \mathbf{i}}=\mathbf{0} .
$$

Observe that the vectors $\mathbf{a}_{\mathbf{i}}, i=\overline{1, n}$, belong to the symmetry axis and the vectors $\mathbf{x}_{2 \mathbf{i}}, i=\overline{1, k}$, are perpendicular on it. The above relation can therefore be fulfilled if and only if each of the two sums vanishes. Interesting for our purpose is the second one:

$$
\sum_{i=1}^{k}\left(m_{2 i-1}-m_{2 i}\right) \mathbf{x}_{2 \mathbf{i}}=\mathbf{0} .
$$

The idea of the proof is to show that relation (2) can be fulfilled for all symmetric centered solutions only if $m_{2 i-1}=m_{2 i}, i=\overline{1, k}$.

Let's suppose that $m_{2 i-1} \neq m_{2 i}$ for every $i \in\{1, \ldots, k\}$. Since $\mathbf{x}_{2 \mathbf{i}}, i=$ $\overline{1, k}$, are collinear vectors, relation $(2)$ is an equality between real numbers, where $x_{2 i}, i=\overline{1, k}$, have all the same sign and therefore the expressions $m_{2 i-1}-m_{2 i}, \quad i=\overline{1, k}$, cannot have all the same sign.

Consider a partition $\{A, B\}$ of the set $\{1, \ldots, k\}$ such that

$$
\left(m_{2 i-1}-m_{2 i}\right) x_{2 i} \geq 0, \quad \text { for } i \in A,
$$

and

$$
\left(m_{2 j-1}-m_{2 j}\right) x_{2 j} \leq 0, \quad \text { for } j \in B \text {. }
$$

It follows that we can write relation (2) as

$$
\sum_{i \in A}\left(m_{2 i-1}-m_{2 i}\right) x_{2 i}+\sum_{j \in B}\left(m_{2 j-1}-m_{2 j}\right) x_{2 j}=0 .
$$

In case $A=\varnothing$ or $B=\varnothing$ we have $m_{2 i-1}-m_{2 i}, \forall i=\overline{1, k}$, and the theorem is proved. Let's therefore suppose that $A \neq \varnothing$ and $B \neq \varnothing$. We will show that there exist symmetric centered solutions of the Equations (1) that do not fulfill relation (3) all along the motion.

Remark. It is obvious that to each $(\mathbf{q}, \mathbf{p})(0)$ there corresponds a unique $(\mathbf{q}, \dot{\mathbf{q}})(0)$ and vice versa. We will therefore talk sometimes, for expository reasons, about $(\mathbf{q}, \dot{\mathbf{q}})(0)$ instead of $(\mathbf{q}, \mathbf{p})(0)$.

In order to reach our goal consider the set

$$
\mathbf{S}=\left\{(\mathbf{q}, \dot{\mathbf{q}})(0) \mid \exists k \in \mathbf{N}, 1 \leq k \leq n / 2 \text { such that } \mathbf{u}^{(\nu)}(0)=\mathbf{0}, \forall \nu \in \mathbf{N}\right\} .
$$

Observe that every $\mathbf{u}^{(\nu)}(0), \nu \in \mathbf{N}$, is constructed using only $\mathbf{q}(0)$ and $\dot{\mathbf{q}}(0)$.

By Corollary 3 , every $\mathbf{r}:=(\mathbf{q}, \dot{\mathbf{q}})(0) \in \mathbf{S}$ leads to a symmetric centered solution $(\mathbf{q}, \dot{\mathbf{q}})$ of the Equations (1). If $\mathbf{r}:=\left(\mathbf{q}_{1}, \ldots, \mathbf{q}_{\mathbf{n}}, \dot{\mathbf{q}}_{1}, \ldots, \dot{\mathbf{q}}_{\mathbf{n}}\right)(0)$, denote

$$
\mathbf{r}_{\gamma}^{\mathrm{ij}}:=\left(\mathbf{q}_{1}, \ldots, \mathbf{q}_{\mathbf{n}}, \dot{\mathbf{q}}_{1}, \ldots, \gamma \dot{\mathbf{q}}_{\mathbf{i}}, \ldots, \gamma \dot{\mathbf{q}}_{\mathbf{j}}, \ldots, \dot{\mathbf{q}}_{\mathbf{n}}\right)(0), \quad \gamma \in \mathbf{R} .
$$


The following result expresses the fact that if initial conditions, leading to a symmetric centered solution, are given and if we change simultaneously (conserving the symmetry) the magnitude or/and the sense of the velocities of two symmetric particles, without changing the direction, then the new initial conditions also lead to a symmetric centered solution.

Lemma 5. If $\mathbf{r} \in \mathbf{S}$ then $\mathbf{r}_{\gamma}^{2 \mathbf{i}-1,2 \mathbf{i}} \in \mathbf{S}, \forall i=\overline{1, k}, \forall \gamma \in \mathbf{R}$.

Proof. Let $\mathbf{u}$ correspond to $\mathbf{r}$ and $\tilde{\mathbf{u}}$ to $\mathbf{r}_{\gamma}^{2 \mathbf{i}-1,2 \mathbf{i}}$. Then, obviously, $\mathbf{u}(0)=$ $\tilde{\mathbf{u}}(0)=\mathbf{0}$. Also observe that

$$
\dot{\tilde{\mathbf{u}}}(0)=\left(\ldots, \gamma\left(\dot{q}_{2 i-1}^{1}-\dot{q}_{2 i}^{1}\right), \gamma\left(\dot{q}_{2 i-1}^{2}+\dot{q}_{2 i}^{2}\right), \ldots\right)(0) .
$$

Since $\dot{\mathbf{u}}(0)=\mathbf{0}$ it follows that

$$
\left(\dot{\mathbf{q}}_{2 i-1}^{1}-\dot{q}_{2 i}^{1}\right)(0)=\left(\dot{q}_{2 i-1}^{2}+\dot{q}_{2 i}^{2}\right)(0)=0
$$

and thus $\dot{\tilde{\mathbf{u}}}(0)=\mathbf{0}$. Analogously, using the fact that $\mathbf{u}^{(\nu)}(0)=\mathbf{0}, \forall \nu \geq 2$, we obtain $\tilde{\mathbf{u}}^{(\nu)}(0)=\mathbf{0}, \forall \nu \geq 2$, and our lemma is proved.

Let's now state a result which shows that a particle of the $n$-body system, having a nonzero initial velocity, firstly moves on the direction given by the velocity vector.

Proposition 6. Consider $(\mathbf{q}, \dot{\mathbf{q}})(0) \in\left(\mathbf{R}^{2 n}-\Delta\right) \times \mathbf{R}^{2 n}$ to be initial conditions of the Equations (1) and $(\mathbf{q}, \mathbf{p})$ the corresponding solution, defined on a maximal interval $\left(t^{-}, t^{+}\right)$. Choose a particle $m_{i}$ and consider a fixed straight line $\Gamma_{i}$ through $m_{i}$ at $t=0$ but such that the velocity vector $\dot{\mathbf{q}}_{\mathbf{i}}(0) \neq \mathbf{0}$ has not $\Gamma_{i}$ as support line. Then there exists $t^{*}>0$, sufficiently small, such that the particle $m_{i}$ belongs, at $t=t^{*}$, to the half-plane containing $\dot{\mathbf{q}}_{\mathbf{i}}(0)$.

Proof. Since for every $t>0$ there exists $\tau_{t} \in(0, t)$ such that

$$
\mathbf{q}_{\mathbf{i}}(t)=\mathbf{q}_{\mathbf{i}}(0)+\dot{\mathbf{q}}_{\mathbf{i}}(0) t+(1 / 2) \mathbf{q}_{\mathbf{i}}\left(\tau_{t}\right) t^{2},
$$

and since we can always choose an interval $(0, t)$ where the motion (and consequently $\mathbf{q}_{\mathbf{i}}$ ) is bounded, the conclusion follows for $t^{*}>0$, sufficiently small.

Consider now $(\tilde{\mathbf{q}}, \dot{\tilde{\mathbf{q}}})(0) \in \mathbf{S}$. Using the property of Lemma 5 a finite number of times, we can obtain $(\mathbf{q}, \dot{\mathbf{q}})(0) \in \mathbf{S}$ with $\dot{\mathbf{q}}(0)$ suitably choosen such that, by Proposition 6,

and

$$
\left(m_{2 i-1}-m_{2 i}\right) x_{2 i}\left(t^{*}\right)>\left(m_{2 i-1}-m_{2 i}\right) x_{2 i}(0), \quad \forall i \in A,
$$

$$
\left(m_{2 j-1}-m_{2 j}\right) x_{2 j}\left(t^{*}\right)>\left(m_{2 j-1}-m_{2 \jmath}\right) x_{2 j}(0), \quad \forall j \in B,
$$

which means that relation (3) fails for the corresponding solution $(\mathbf{q}, \mathbf{p})$ at time $t^{*}$. Therefore, for at least one $i \in\{1, \ldots, k\}$, we have $m_{2 i-1}=m_{2 i}$. Iterating the above process, after a finite number of steps, we obtain

$$
m_{2 i-1}=m_{2 i} \quad \text { for all } i \in\{1, \ldots, k\}
$$

and Theorem 4 is proved. 
Remark. Apparently this proof does not make obvious that dynamics is essential. Actually, relation (3) is derived from the fact that the solutions are restricted to the invariant set $\mathbf{Q} \times \mathbf{P}$, restriction which is possible by the existence of the momenta and center of mass integrals. These first integrals are clearly consequences of the dynamics (even for the more general attraction law of the inverse $(\alpha+1)$-power of the distance, with $\alpha>0)$. Also observe that for $n=4$ the equality of the symmetric masses follows directly from the equations of motion. We have seen in [4] that this fact can be proved (also in the spatial problem) without having to impose the condition that the center of mass of the system lies on the symmetry axis. Hitherto we could not extend this method to the general case and it seems that a direct algebraic attack on the equations of motion is unsucessful even in the symmetric centered case.

\section{IMPROBABILITY OF SYMMETRIC SOLUTIONS}

In some previous papers (see $[1,2,3,5]$ ) we have studied the sets of initial conditions leading to some special solutions of the $n$-body problem (rectilinear, collinear, flat, syzygy) from the measure theory and topological point of view. Excepting the set of initial conditions leading to syzygy solutions in the planar 3-body problem (which is nonvoid and open) all the others are of measure zero and nowhere dense. Using the same principle we will prove the following result that actually states that symmetric centered solutions are very improbable.

Theorem 7. The set $\mathbf{D}$ of initial conditions leading to symmetric centered solutions in the planar n-body problem (general) measure zero and nowhere dense relative to the set $\left(\mathbf{R}^{2 n}-\Delta\right) \times \mathbf{R}^{2 n}$ (with the induced topology) of all initial conditions that define solutions in the considered plane.

Proof. In order to prove the first part observe that

$$
\mathbf{u}(0)=\dot{\mathbf{u}}(0)=\mathbf{0}
$$

represents $2 n$ scalar, independent relations that must be fulfilled by the initial conditions leading to a symmetric centered solution. Thus the dimension of $\mathbf{D}$ in $\left(\mathbf{R}^{2 n}-\Delta\right) \times \mathbf{R}^{2 n}$ (which dimension is $4 n$, see [1]) is at most $4 n-2 n=2 n$ and the conclusion concerning the measure theory point of view follows.

Suppose now that int $\overline{\mathbf{D}} \neq \varnothing$. Since int $\overline{\mathbf{D}}$ is open, it follows that there exists another open set $\mathbf{V}$, such that $\mathbf{V} \subset \operatorname{int} \overline{\mathbf{D}} \subset \overline{\mathbf{D}}$. As $\mathbf{D} \subset \overline{\mathbf{D}}$ but $\overline{\mathbf{D}} \not \subset \mathbf{D}$ (because if this is not the case then $\mathbf{D}=\overline{\mathbf{D}}$ and using the fact that $\mathbf{V}=\operatorname{int} \mathbf{V} \subset \overline{\mathbf{D}}=\mathbf{D}$ it would follow that $\mathbf{D}$ is not of measure zero, contradicting the above proof), the existence of some $\mathbf{s}=(\mathbf{q}, \mathbf{p})(0) \in \overline{\mathbf{D}}$ such that $\mathbf{s} \notin \mathbf{D}$, follows. Therefore the corresponding solution of the Equations (1), defined on $\left(t^{-}, t^{+}\right)$, is not symmetric centered. It means that, for each $k \in \mathbf{N}, 1 \leq k \leq n / 2$, every corresponding $\mathbf{u}$ fulfills the condition that there exists $t_{\mathbf{u}} \in\left(t^{-}, t^{+}\right)$such that $\mathbf{u}\left(t_{\mathbf{u}}\right) \neq \mathbf{0}$. (By every $\mathbf{u}$ we understand that all possibilities of renumbering the bodies are taken into account.) Thus, for every $\mathbf{u}$ we can say that there exists $i \in\{1, \ldots, n\}$ such that $\mathbf{u}_{\mathbf{i}}\left(t_{\mathbf{u}}\right) \neq \mathbf{0}$. In case $i \in\{1, \ldots, 2 k\}$ then, if 
$i=2 j-1$, it follows that $q_{2 j-1}^{1}\left(t_{\mathbf{u}}\right) \neq q_{2 j}^{1}\left(t_{\mathbf{u}}\right)$ and if $i=2 j$ then $q_{1 j-1}^{2}\left(t_{\mathbf{u}}\right) \neq$ $-q_{2 j}^{2}\left(t_{\mathbf{u}}\right)$. For $i \in\{2 k+1, \ldots, n\}$ we have $q_{i}^{2}\left(t_{\mathbf{u}}\right) \neq 0$.

By the theorem of continuity of the solution with respect to initial data we have: $\forall \varepsilon>0, \forall I \subset\left(t^{-}, t^{+}\right), I$ compact, there exists a $\tilde{\mathbf{v}}$, a neighborhood of $(\mathbf{q}, \mathbf{p})(0)$ such that, for every $(\tilde{\mathbf{q}}, \tilde{\mathbf{p}})(0) \in \tilde{\mathbf{V}}$, the corresponding solution $(\tilde{\mathbf{q}}, \tilde{\mathbf{p}})$ is defined on $I$ and

$$
|(\tilde{\mathbf{q}}, \tilde{\mathbf{p}})(t)-(\mathbf{q}, \mathbf{p})(t)|<\varepsilon, \quad \forall t \in I .
$$

It is now easy to see that, considering each of the above cases, for $\varepsilon>0$ sufficiently small, every $\tilde{\mathbf{u}}$ of the corresponding $(\tilde{\mathbf{q}}, \tilde{\mathbf{p}})$ solution, defined by $(\tilde{\mathbf{q}}, \tilde{\mathbf{p}})(0) \in \tilde{\mathbf{V}}$, fulfills the condition $\tilde{\mathbf{u}}\left(t_{\mathbf{u}}\right) \neq \mathbf{0}$. Since there exists an open set $\tilde{\mathbf{V}}$ of initial conditions leading to nonsymmetric centered solution, with $\mathbf{s} \in \tilde{\mathbf{V}}$, the existence of $\mathbf{s} \in \overline{\mathbf{D}}$, with $s \notin \mathbf{D}$, is contradicted. Thus $\operatorname{int} \bar{D}=\varnothing$ and the theorem is proved.

Observe that Theorem 7 is also true if we consider a symmetric (not necessarily centered) spatial solution of the $n$-body problem. We have chosen this way to present the facts because of expository reasons.

Note finally that all results work for the more general law of the inverse $(\alpha+1)$-power of the distance, $\alpha>0$.

\section{ACKNOWLEDGMENT}

The author is indebted to Dr. Christian Klingenberg for valuable comments concerning the manuscript.

\section{REFERENCES}

1. F. N. Diacu, Improbability of collinear solutions in the $n$-body problem with generalized attraction law, Astron. Nachr. 309 (1988), 233-236.

2. __ Wintner's collinear and flat solutions are nowhere dense, Celest. Mech. 44 (1988), 261-265.

3. _ On the planar syzygy solutions of the 3-body problem, Celest. Mech. (to appear).

4. _ . The masses in a symmetric solution of the four body problem, Celest. Mech. (to appear).

5. F. N. Diacu and Á. Pál, Improbability of some special solutions in the $n$-body problem with generalized attraction law, Babes-Bolyai Univ., Fac. Math. Res. Sem. 10 (1987), 19-24.

6. A. Wintner, The analytical foundations of celestial mechanics, Princeton Univ. Press, Princeton, NJ, 1941.

SONDERForsChUNGSBereich 123, Universität Heidelberg, InStitut Für ANGEWANDTE Mathematik, Im Neuenheimer Feld 294, 6900 Heidelberg 1, Federal Republic of GerMANY 